\title{
An Unexpected Favourable Evolution of Advanced Stage Cirrhosis
}

\author{
Tiago Rabadão ${ }^{1}$, Leonor Naia ${ }^{1}$, Mariana Teixeira ${ }^{1}$, Marcelo Aveiro $^{1}$, Filipa Ferreira ${ }^{1}$, Margarida Eulálio ${ }^{1}$, Suzana Calretas $^{2}$ \\ ${ }^{1}$ Internal Medicine Department, Centro Hospitalar do Baixo Vouga, Aveiro, Portugal \\ ${ }^{2}$ Adult Liver Transplant Unit, Centro Hospitalar e Universitário de Coimbra, Coimbra, Portugal
}

Received: 04/11/2020

Accepted: $12 / 11 / 2020$

Published: 03/12/2020

How to cite this article: Rabadão T, Naia L, Texeira M, Aveiro M, Ferreira F, Eulálio M, Calretas S. An unexpected favourable evolution of advanced stage cirrhosis. EJCRIM 2020;7: doi:10.12890/2020_002122.

Conflicts of Interests: The Authors declare that there are no competing interests.

This article is licensed under a Commons Attribution Non-Commercial 4.0 License

\section{ABSTRACT}

The elimination of the aetiological factors causing liver injury is an important cornerstone in preventing progression and increasing survival in patients with cirrhosis. The authors present the case of a 63-year-old woman with a history of long-term alcohol abuse and consequent liver cirrhosis. Over the years, the patient presented progressive deterioration with severe malnutrition and had multiple hospital admissions due to decompensated cirrhosis, including refractory ascites, variceal bleeding and an extensive portal vein thrombosis (PVT). Anticoagulant therapy was not initiated due to a high risk of variceal bleeding. She eventually became abstinent, but PVT precluded a liver transplant. Over the following 10 years, her performance status gradually improved, with no new decompensation episodes and liver function normalization, although refractory ascites persisted. Abdominal CT showed spontaneous recanalization of the portal vein and a transjugular intrahepatic portosystemic shunt (TIPS) procedure was performed with gradual improvement of ascites. In this atypical case, an unexpected favourable evolution of advanced stage cirrhosis was observed with long-term improvement in clinical status and liver function, resulting in an estimated 10 -year cumulative mortality rate of $99.98 \%$ and highlighting the importance of abstinence. Unexpectedly, spontaneous complete repermeabilization of the PVT was also observed, despite its extent and the absence of anticoagulation therapy.

\section{LEARNING POINTS}

- The elimination of the aetiological factors causing liver injury is the cornerstone in preventing decompensation and improving prognosis in patients with cirrhosis.

- Spontaneous complete recanalization of the portal vein may occur.

- A transjugular intrahepatic portosystemic shunt (TIPS) is effective in managing some of the major complications of portal hypertension, such as refractory ascites, and provides secondary prophylaxis of oesophageal variceal haemorrhage.

\section{KEYWORDS}

Decompensated cirrhosis, alcohol abstinence, portal vein thrombosis, refractory ascites, transjugular intrahepatic portosystemic shunt (TIPS)

\section{INTRODUCTION}

Cirrhosis is an important cause of morbidity and mortality worldwide. In the past, it was considered an irreversible state, but multiple clinical studies have shown regression of liver fibrosis and reversal of cirrhosis ${ }^{[1]}$. The elimination of the aetiological factors causing liver injury, including alcohol consumption, is an important cornerstone in its management, and clearly effective in preventing decompensation which is associated with high mortality rates, and also in increasing the survival of patients with compensated cirrhosis ${ }^{[2]}$. However, in decompensated cirrhosis it is less effective and depends on the status of the liver disease ${ }^{[3]}$. 


\section{CASE DESCRIPTION}

A 63-year-old white woman presented with alcohol-related liver disease diagnosed 15 years previously. She had a significant medical history of long-term chronic alcohol abuse ( $90 \mathrm{~g} /$ day), arterial hypertension, dyslipidemia and hyperuricemia; no history of blood products transfusion, illicit drug injection, or other liver disease risk factors; and no medical follow-up. Five years after her earlier diagnosis, following hospital admission for traumatic brain injury, the patient initiated regular medical monitoring. By that time, she was Child-Pugh class B (8 points) with a MELD score of 13. Liver study excluded other viral, autoimmune and metabolic aetiologies. During the following 2 years, the patient experienced clinical deterioration with severe malnutrition, a performance status of 4 and multiple hospital admissions due to decompensation including refractory ascites (Fig. 1), recurrent hepatic encephalopathy, variceal bleeding (oesophageal and rectal) and hyponatremia, and also infections (sepsis and spontaneous bacterial peritonitis).

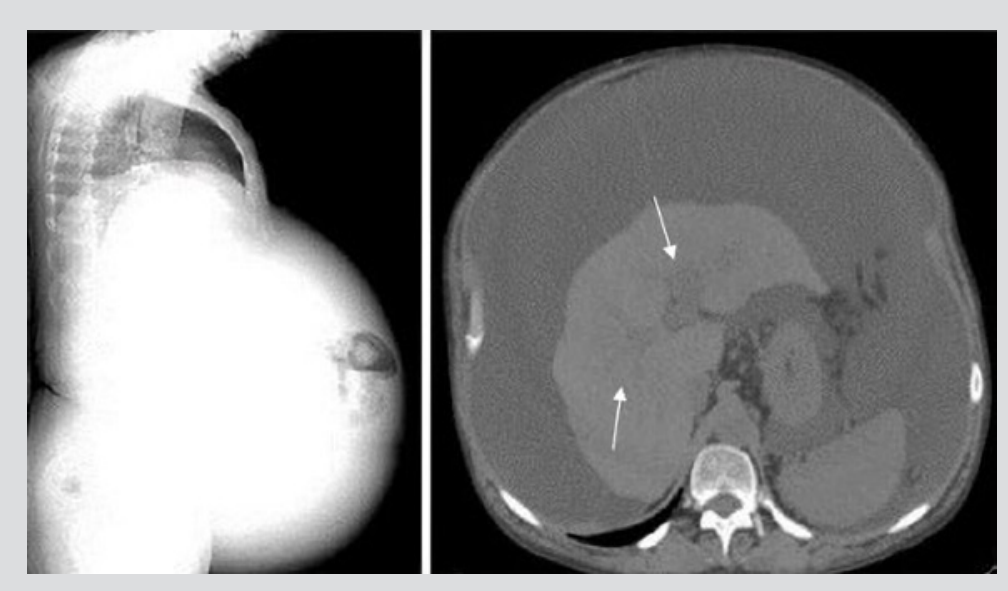

Figure 1. Abdominal CT showing a cirrhotic liver with no focal

lesions, a large volume of ascites and a patent portal vein (arrows)

Abdominal computed tomography (CT) showed portal vein thrombosis (PVT) (>50\% of the lumen) that reached the left and right intrahepatic branches (Fig. 2). The coagulation tests excluded thrombophilia. Anticoagulant therapy was not initiated due to the high variceal bleeding risk. At that time, the patient stopped alcohol consumption, and a pre-liver transplant study was carried out. Unfortunately, the patient was not elected for transplantation due to the extent of PVT (including the superior mesenteric vein) (Fig. 3).

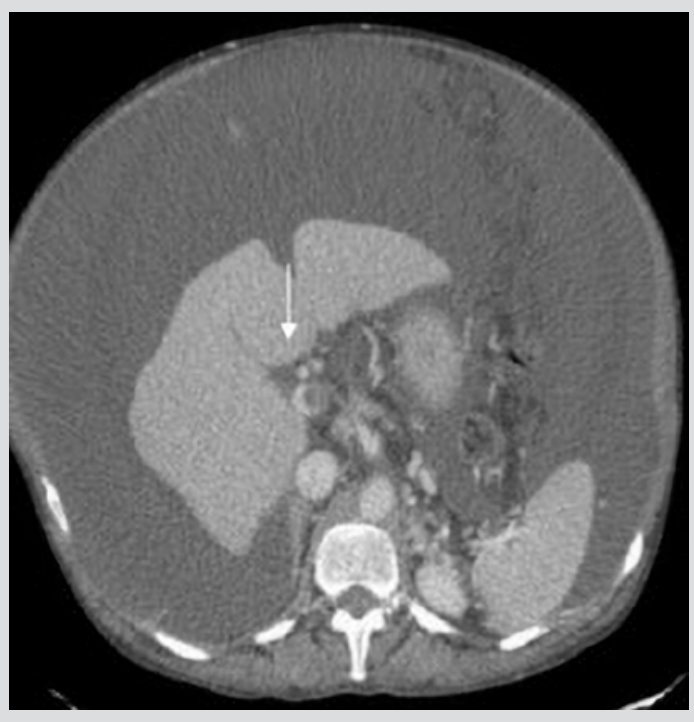

Figure 2. Abdominal CT showing portal vein thrombosis (PVT) $(>50 \%$ of the lumen) extending to the left and right intrahepatic branches (arrow)

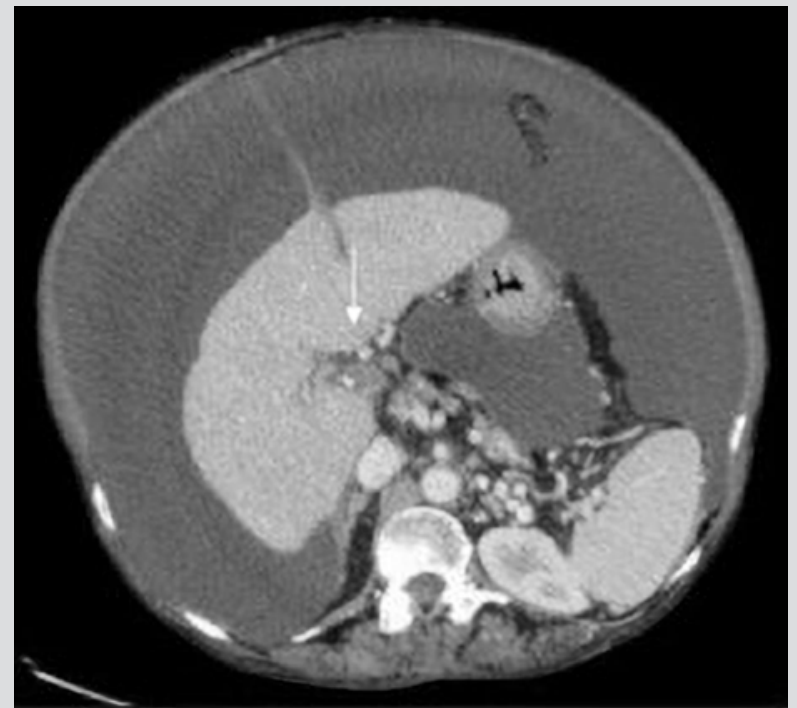

Figure 3. Abdominal CT, a few months later, showing complete portal vein thrombosis (PVT) (arrow) 
The patient maintained regular follow-up, and continued alcohol abstinence. Her neurological and performance status gradually improved and she showed no new decompensations. However, refractory ascites persisted, with regular large volume paracentesis needed. Five years later, abdominal CT showed spontaneous complete recanalization of the portal vein (Fig. 4).

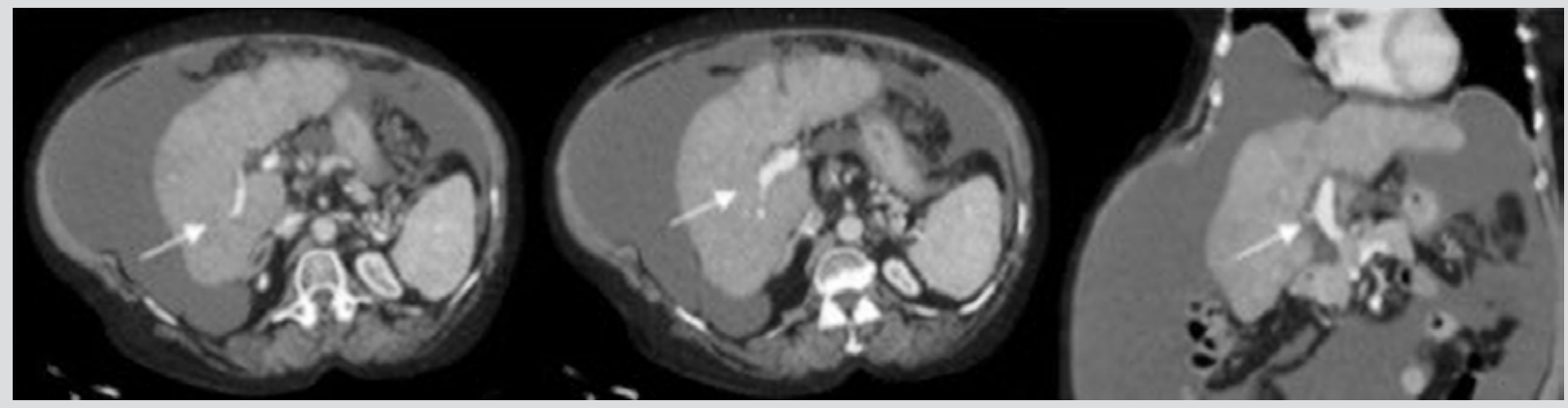

Figure 4. Abdominal CT showing total recanalization of the portal vein (arrows)

At this time, the patient had a performance status of 1 and a MELD score of 7 and was Child-Pugh class B (7 points). A transjugular intrahepatic portosystemic shunt (TIPS) procedure was performed with gradual improvement of ascites. Currently, the patient has a performance status of 0 , and she is Child-Pugh class A ( 5 points) with a MELD score of 7 . She has no ascites, is not taking any diuretics and has a patent TIPS. She maintains close hepatology follow-up.

\section{DISCUSSION}

The clinical course of cirrhosis is asymptomaticuntil increasing portal pressure and worsening liver function result inclinical decompensation ${ }^{[3]}$. This report highlights the possibility of reversing this clinical course, despite previous severe decompensations, through the elimination of factors causing liver injury, thus improving the patient's quality of life and prognosis. In this case, an unexpected favourable evolution of advanced stage cirrhosis was observed, with an estimated 10-year cumulative mortality rate of $99.98 \%$. Long-term improvement in clinical status and liver function has resulted in the current mortality rate of $3.4 \%$ per year, demonstrating the importance and impact of abstinence from alcohol use on clinical outcome. Currently, the patient maintains preserved liver function, without any indication for liver transplant. We also observed spontaneous complete repermeabilization of the portal venous system, despite the extent of thrombosis and lack of anticoagulation therapy due to a high risk of variceal bleeding.

PVT is a common complication of advanced liver disease and hepatobiliary malignancies, caused by underlying multifactorial prothrombotic states ranging from asymptomatic to life-threating conditions related to portal hypertension and hepatic decompensation. On the other hand, PVT has been shown to be independently associated with a higher risk of variceal bleeding, leading to increased mortality. Studies also demonstrate increased mortality associated with thrombus extension into the superior mesenteric vein. Although spontaneous complete recanalization of the portal vein may occur, mainly when thrombosis is partial ${ }^{[4]}$, treatment with anticoagulants, once considered contraindicated, is now indicated.

The authors want to highlight the challenges of anticoagulant therapy in patients with cirrhosis: their case complexity, with frequent recurrent bleeding episodes and uncontrolled disease, and the lack of clinical trials with this type of patient, possibly encourage noninitiation of such therapy. The safer anticoagulants, the potential role of direct oral anticoagulants and the optimal duration of therapy are still a matter of debate ${ }^{[5]}$.

All studies underline the absolute need for screening for gastroesophageal varices prior to anticoagulant therapy, so that primary or secondary bleeding preventive strategies can be initiated. In contrast to the case presented, PVT may no longer be a contraindication to liver transplantation depending on its extent. Due to refractory ascites, TIPS was the chosen treatment, since liver function was preserved and consequently a liver transplant was not necessary. This procedure helped improve the patient's condition. The patient maintains close follow-up with screening for hepatocellular carcinoma and other complications, such as recurrence of thrombosis. 


\section{REFERENCES}

1. Zhou W, Zhang Q, Qiao L. Pathogenesis of liver cirrhosis. World J Gastroenterol 2014;20(23):7312-7324.

2. D’Amico G, Garcia-Tsao G, Pagliaro L. Natural history and prognostic indicators of survival in cirrhosis: a systematic review of 118 studies. J Hepatol $2006 ; 44: 217-231$.

3. European Association for the Study of the Liver. EASL Clinical Practice Guidelines: Management of decompensated cirrhosis. J Hepatol 2018; https://doi.org/10.1016/j. jhep.2018.03.024.

4. European Association for the Study of the Liver. EASL Clinical Practice Guidelines: Vascular diseases of the liver. J Hepatol 2016;64:179-202

5. Faccia M, Ainora ME, Ponziani FR, Riccardi L, Garcovich M, Gasbarrini A, et al. Portal vein thrombosis in cirrhosis: why a well-known complication is still matter of debate. World J Gastroenterol 2019;25(31):4437-4451. 